\title{
Heterocyclic aromatic amide protecting groups for aryl and phthalocyaninesulfonic acids
}

\author{
Zhaopeng Li, Johan van Lier, and Clifford C. Leznoff
}

\begin{abstract}
Pyrroles, indole, imidazole, and a pyrazole were treated with 3,4-dibromobenzenesulfonyl chloride to form 3,4-dibromobenzenesulfonamides. The 1-(3,4-dibromophenylsulfonyl)pyrrole and 1-(3,4-dibromophenylsulfonyl)indole were stable to $\mathrm{CuCN}$ in DMF to produce 1-(3,4-dicyanophenylsulfonyl)pyrrole and 1-(3,4-dicyanophenylsulfonyl)indole, which upon treatment with ammonia in 2- $N, N$-dimethylaminoethanol gave the protected phthalocyanine-2,9,16,23tetrasulfonamides. Base cleavage of these sulfonamides yielded the free acids. A mixed condensation of 4,5-diheptylphthalonitrile and 1-(3,4-dicyanophenylsulfonyl)pyrrole gave 9,10,16,17,23,24-hexakis(1-heptyl)-2-(1pyrrolylsulfonyl)phthalocyanine. Cleavage of the latter yielded the lithium salt of the monosulfonic acid.
\end{abstract}

Key words: sulfonic acid blocking groups, phthalocyanine sulfonic acids, 1-(3,4-dicyanophenylsulfonyl)pyrrole, I-(3,4-dicyanophenylsulfonyl)indole.

Résumé : La réaction du chlorure de 3,4-dibromobenzènesulfonyle avec des pyrroles, l'indole, l'imidazole et un pyrazole conduit à la formation de 3,4-dibromobenzènesulfonamides. Le 1-(3,4-dibromophénylsulfonyl)pyrrole et du 1-(3,4-dibromophénylsulfonyl)indole ont été soumis à l'action du CuCN dans le DMF pour produire du 1-(3,4-dicyanophénylsulfonyl)pyrrole et du 1-(3,4-dicyanophénylsulfonyl)indole qui, par traitement avec de l'ammoniac dans le 2-N,N-diméthylaminoéthanol conduit aux phtalocyanine-2,9,16,23-tétrasulfonamides protégés. Le clivage basique de ces sulfonamides conduit aux acides libres. Une condensation mixte du 4,5-diheptylphtalonitrile et du 1-(3,4-dicyanophénylsulfonyl)pyrrole conduit à la $9,10,16,17,23,24$-hexakis(1-heptyl)-2-(1-pyrrolylsulfonyl)phtalocyanine. Le clivage de cette dernière foumit le sel de lithium de l'acide monosulfonique.

Mots clés : acide sulfonique comme agents bloqueurs, acides phtalocyanine sulfoniques, 1-(3,4-dicyanophénylsulfonyl)pyrrole, 1-(3,4-dicyanophénylsulfonyl)indole.

[Traduit par la Rédaction]

\section{Introduction}

Phthalocyanine $(\mathrm{Pc})$ sulfonic acids have potential as photodynamic therapy (PDT) agents of cancer tumours (1, 2). Although phthalocyanine -2,9,16,23-tetrasulfonic acid is commercially available as a mixture of isomers, it has been shown that phthalocyanine-2-sulfonic acid and phthalocyanine2,9-disulfonic acid are more effective for direct cell kill (3-9). Unfortunately, only small samples obtained by high-performance liquid chromatography (HPLC) of these unsymmetrical phthalocyanine sulfonic acids $(6-8)$ were available for PDT studies $(9,10)$. Some recent attempts at preparing mono sulfonated Pes by the oxidation of sulfides (11) or trisulfonated Pcs $(12,13)$ via subphthalocyanines $(14,15)$ gave Pcs in low yield.

One roadblock in the development of a rich variety of readily available sulfonated Pcs is the paucity of suitable blocking groups or precursors of sulfonic acids. Sulfonamides are common compounds made from sulfonyl chlorides and amines, and sulfonamides are widely used as blocking groups of amines. Cleavage (16) of sulfonamides to recover the free amines is not a trivial operation, but a large number of sophisticated procedures (17) have been used to accomplish this task, often requiring harsh conditions such as the use of concentrated acid and high temperatures (18), or reducing agents (19). In most of these procedures little attention is paid to the sulfonic acid residue remaining after the cleavage, and indeed, the sulfonic acid group is often transformed in the process (17-19). The recent description of a neopentyl ester group as a blocking group (20) of sulfonic acid seemed interesting to us but early experiments indicated it to be unsuitable for phthalocyanine synthesis.

Aryl sulfonyl chlorides have been used as blocking groups of five-membered ring aromatic amines such as imidazole, $(21,22)$, pyrrole $(23)$, and indole $(24)$, and these were cleaved under a variety of conditions, liberating both the amine and recovered sulfonic acids, and hence we directed our attention to the use of these five-membered heterocyclic amines as blocking groups of aryl sulfonic acids. It proved to be essential to develop an aryl sulfonic acid blocking group that was stable to both phthalonitrile and phthalo-

Z. Li and C.C. Leznoff. ${ }^{1}$ Department of Chemistry, York University, Toronto, ON M3J IP3 Canada.

J. van Lier. MRC Group in the Radiation Sciences, Faculty of Medicine, Université de Sherbrooke, PQ JIN 5N4, Canada.

'Author to whom correspondence may be addressed. Telephone: (416) 736-2100, ext. 33838. Fax: (416) 736-5936.

e-mail: leznoff@yorku.ca 
cyanine formation and yet could be cleaved under conditions in which the sulfonic acid groups remained intact.

\section{Results and discussion}

Chlorosulfonation of 1,2-dibromobenzene (1) readily gave 3,4-dibromobenzenesulfonyl chloride (2) (25). Solov'eva et al. (25) described the synthesis of a wide variety of $N, N$-dialkyl-3,4-dibromobenzenesulfonamides for use in phthalonitrile and phthalocyanine synthesis, but these sulfonamides are not readily cleaved to the free acids. We first decided to try to see if aryl groups such as diphenylamine (3) used in making $N, N$-diphenylbenzenesulfonamide would be useful in phthalocyanine synthesis and at the same time compare that analog with a series of heteroarylbenzenesulfonamides derived from pyrrole (4), 2-ethylpyrrole (5), 3,5-dimethylpyrazole (6), imidazole (7), and indole (8).

Treatment of sulfonyl chloride 2 with 3 in refluxing $\mathrm{CHCl}_{3}$ and pyridine (py) afforded 3,4-dibromo- $N, N$-diphenylbenzenesulfonamide (9) in high yield. On the other hand, pyrroles 4 and 5 were first treated with $\mathrm{NaH}$ in tetrahydrofuran (THF) to form the sodium derivatives, which then reacted with $\mathbf{2}$ at room temperature to give 1-(3,4-dibromophenylsulfonyl)pyrrole (10), 1-(3,4-dibromophenylsulfonyl)-2-ethylpyrrole (11), and 1-(3,4-dibromophenylsulphonyl)-3,5-dimethylpyrazole (12) in 50,3, and $84 \%$ yields, respectively. The reaction of imidazole (7) and indole (8) with base in $\mathrm{CHCl}_{3}$ likewise gave 1-(3,4-dibromophenylsulfonyl)imidazole (13) and 1-(3,4- dibromophenylsulfonyl)indole (14) in 34 and $50 \%$ yields, respectively (Scheme 1). The low yield of $\mathbf{1 1}$ precluded further work on this compound, although we had envisioned that the ethyl group of 5 would afford enhanced solubility to the subsequent phthalonitrile and phthalocyanine.

At this point it was important to test the stability of sulfonamides $9, \mathbf{1 0}$, and 12-14 to the conditions of $(i)$ the Rosenmund - von Braun reaction (CuCN, $200^{\circ} \mathrm{C}$, DMF) (26), (ii) phthalocyanine formation (MOR or $\mathrm{NH}_{3}$ ), and (iii) sulfonamide cleavage. Sulfonamides $9,10,13$, and 14 were stable to the Rosemund - von Braun reaction, but 12 was not and was abandoned for further consideration. Sulfonamides 9,10 , and 14 were stable to $\mathrm{NaOCH}_{3}$ at room temperature and $\mathrm{NH}_{3}$ in 2-N,N-dimethylaminoethanol (DMAE), possible methods for use in phthalocyanine formation but 13 did not survive these conditions (Table 1) and was dropped as a potential candidate for $\mathrm{Pc}$ formation. Sulfonamides $\mathbf{1 0}$ and 14 were solvolyzed by $\mathrm{NaOCH}_{3}$ in refluxing methanol to give 3,4-dibromophenylsulfonic acid (15) (27), but again 12 and 13 gave 15 simply upon stirring at room temperature as above (Table 1). All sulfonamides were stable to concentrated $\mathrm{HCl}$ at room temperature. Thus, only sulfonamides 9 , 10, and 14 were suitable substrates for development for phthalocyanine synthesis.

The dibromosulfonamides 9,10 , and 14 were readily converted via CuCN in DMF to 3,4-dicyano- $N, N$-diphenylbenzenesulfonamide (16), 1-(3,4-dicyanophenylsulfonyl)pyrrole (17), and 1-(3,4-dicyanophenylsulfonyl)indole (18) in 29, 43, and $45 \%$ yields, respectively (Scheme 2). The $N, N$-diphenylsulfonamide 16 readily formed $N, N, N^{\prime}, N^{\prime}, N^{\prime \prime}, N^{\prime \prime}, N^{\prime \prime \prime}, N^{\prime \prime \prime}$. octaphenylphthalocyanine-2,9,16,23-tetrasulfonamide (19) in $50 \%$ yield when treated with lithium in DMAE at $70^{\circ} \mathrm{C}(28)$.
Scheme 1.<smiles>Brc1ccccc1Br</smiles>

1<smiles>O=S(=O)(O)c1ccc(Br)c(Br)c1</smiles>

15<smiles>[R10]Nc1ccc(OS(=O)(=O)[O-])cc1Br</smiles><smiles>[R16]S(=O)(=O)c1ccc(Br)c(Br)c1</smiles>

2

$3 \mathrm{R}=\mathrm{Ph}$<smiles>[R16]c1ccccc1</smiles>

$9 \mathrm{R}=\mathrm{Ph}$<smiles></smiles><smiles>[R2]c1ccsc1CC</smiles><smiles></smiles><smiles>[R20]C[18F]</smiles><smiles>[Mg]</smiles><smiles>CSC=N</smiles><smiles>[R2][Y20]1cccn1</smiles><smiles></smiles><smiles></smiles><smiles>[R16]=c1ccc2c(c1)C=CC=2</smiles>

Since we had already shown that 10 and 14 are cleaved by metal alkoxides at refluxing methanol conditions, we treated 17 and 18 with $\mathrm{NH}_{3}$ in DMAE to form $N, N^{\prime}, N^{\prime \prime}, N^{\prime \prime \prime}$. tetrakis-(1-pyrrolyl)phthalocyanine-2,9,16,23-tetrasulfonamide (20) and $N, N^{\prime}, N^{\prime \prime}, N^{\prime \prime \prime}$-tetrakis-(1-indolyl) phthalocyanine-2,9, 16,23tetrasulfonamide $(21)$ in 33 and $42 \%$ yields, respectively. Under similar conditions to the formation of 21 and 22 , but with the addition of $\mathrm{Zn}(\mathrm{OAc})_{2}, 17$ and 18 were converted directly to $N, N^{\prime}, N^{\prime \prime}, N^{\prime \prime \prime}$-tetrakis- (1-pyrrolyl)phthalocyanine-2,9,16, 23-tetrasulfonamide zinc(II) (22) and $N, N^{\prime}, N^{\prime \prime}, N^{\prime \prime \prime}$-tetrakis(1-indolyl)phthalocyanine-2, 9,16, 23-tetrasulfonamide zinc(II) (23) in 32 and $68 \%$ yields, respectively (Scheme 2).

As expected Pc 19 proved to be resistant to solvolysis, but Pc sulfonamides 20 and $\mathbf{2 1}$ were readily converted to phthalocyanine-2,9,16,23-tetrasulfonic acid (24) by treatment with lithium 2-N,N-dimethylaminoethoxide in DMAE (for 20) or lithium methoxide in THF-MeOH for 21 (Scheme 2).

Early experiments towards the synthesis of a phthalocyanine monosulfonic acid via a mixed condensation of 17 and phthalonitrile gave insoluble residues difficult to separate by chromatography. To increase the solubility of the 
Table 1. Synthesis of 3,4-dibromosulfonamides and their stability to bases and the Rosenmund - von Braun reaction.

\begin{tabular}{|c|c|c|c|c|c|}
\hline \multirow[b]{2}{*}{ Compound } & \multirow[b]{2}{*}{$\begin{array}{l}\text { Method } \\
\text { (base/solvent) }\end{array}$} & \multirow[b]{2}{*}{ Yield (\%) } & \multicolumn{3}{|c|}{ Stability at $20^{\circ} \mathrm{C}$} \\
\hline & & & $\mathrm{NaOMe}$ & $\mathrm{NH}_{3}$ & $\mathrm{CuCN}-\mathrm{DMF}^{a}$ \\
\hline 9 & $\mathrm{py} / \mathrm{CHCl}_{3}$ & 65 & Stable & Stable & Stable \\
\hline 10 & $\mathrm{NaH} / \mathrm{THF}$ & 50 & Stable ${ }^{b}$ & Stable & Stable \\
\hline 12 & $\mathrm{NaH} / \mathrm{THF}$ & 84 & Unstable & Stable & 一 \\
\hline 13 & $\mathrm{Na}_{2} \mathrm{CO}_{3} / \mathrm{CHCl}_{3}{ }^{e}$ & 34 & Unstable & Unstable & $-\cdots$ \\
\hline 14 & $\mathrm{NaOH} / \mathrm{CH}_{2} \mathrm{Cl}_{2}$ d & 57 & Stable $^{b}$ & Stable & Stable \\
\hline
\end{tabular}

phthalocyanine product, 17 was condensed with 4,5diheptylphthalonitrile (25) (29), as for the formation of $\mathbf{2 2}$, to give 9,10,16,17,23,24-hexakis(1-heptyl)phthalocyanine2 -( $N$-pyrrolyl)sulfonamide (26) in $14.7 \%$ yield. Treatment of 26 with $\mathrm{Zn}(\mathrm{OAc})_{2}$ in DMF readily afforded 9,10,16,17, 23, 24-hexakis(1-heptyl)phthalocyanine-2-( $N$-pyrrolyl)sulfonamide zinc(II) (27) in $86 \%$ yield. Hydrolysis of 27 with lithium methoxide in methanol afforded lithium $9,10,16,17,23,24$ hexakis(1-heptyl)phthalocyanine-2-sulfonate zinc(II) (28) in $85 \%$ yield (Scheme 3 ).

Spectral data of all new compounds were consistent with their structures (see Experimental). Pcs 19-23 exhibited multiplets in their NMR spectra, consistent with the fact that they exist as mixtures of isomers. Mass spectra of all sulfonamides exhibited ions showing loss of $\mathrm{SO}_{2}$ and peaks representing both fragments (30).

We have demonstrated that indole and pyrrole moieties can be successfully used to form sulfonamides as blocking groups in the synthesis of phthalocyanine sulfonic acids.

\section{Experimental}

All organic solvents were dried by appropriate methods and distilled before use. All reagents were freshly distilled, or were recrystrallized and then dried under reduced pressure, before use. Unless otherwise noted, magnetic stirring methods under an inert atmosphere (Matheson High Purity argon) were utilized during distillation or reaction processes, and round-bottom glass vessels chosen such that the quantity of reagents and solvent did not exceed half of the available volume. Water-cooled condensers were used if reaction processes were held near, or at, reflux conditions. Melting points were determined using a Kofler hot-stage melting point apparatus and are reported uncorrected. Infrared spectroscopy was performed on either a Pye Unicam SP3-2000 or a Perkin Elmer 1310 infrared spectrophotometer and FTIR spectroscopy was performed on a Unicam Matheson 3000 FTIR spectrometer, using samples prepared as $\mathrm{KBr}$ discs unless otherwise noted. Mass spectral analyses were performed by Dr. B. Khouw (York University, Toronto, Ontario), Dr. R. Smith (McMaster University, Hamilton, Ontario), and Dr. R.N. Cerny (Midwest Center for Mass Spectrometry, University of Nebraska-Lincoln, Lincoln, Nebraska). HRMS were preformed by Dr. R. Smith (McMaster University, Hamilton, Ontario). Nuclear magnetic resonance
(NMR) spectroscopy was performed at $295-300 \mathrm{~K}$ unless otherwise noted, using a Bruker ARX 400 high-field Fourier transform instrument. Chemical shifts are reported in parts per million relative to a tetramethylsilane (TMS) internal standard.

\section{3,4-Dibromobenzenesulfonyl chloride (2)}

To a flask containing 1,2-dibromobenzene (1) (20 g, $88.47 \mathrm{mmol})$ and dry $\mathrm{CHCl}_{3}(120 \mathrm{~mL})$, which was cooled to ca. $-10^{\circ} \mathrm{C}$ by an ice- $\mathrm{NaCl}$ bath, was added chlorosulfonic acid $(30 \mathrm{~g})$ over $20 \mathrm{~min}$. The light brown solution was stirred at room temperature for $20 \mathrm{~h}$. The reaction mixture turned red during this period. The solution was refluxed for $1 \mathrm{~h}$. After cooling to room temperature, the vigorously stirred reaction mixture was poured onto crushed ice $(200 \mathrm{~g})$. The aqueous and organic layers were separated. The former was washed with $\mathrm{CH}_{2} \mathrm{Cl}_{2}(100 \times 2 \mathrm{~mL})$. The combined organic layer was washed with aqueous $\mathrm{NaHCO}_{3}(10 \%, 150 \mathrm{~mL})$ followed by water until $\mathrm{pH}$ neutral. After drying over $\mathrm{MgSO}_{4}$, and evaporating the solvent, vacuum distillation afforded a colourless solid product $\left(120-125^{\circ} \mathrm{C} / 5 \mathrm{mmHg}\right.$ $\left(1 \mathrm{mmHg}=133.3 \mathrm{~Pa}\right.$ ), $22.7 \mathrm{~g}, 85 \%$ ), mp $3 \mathrm{I}-33^{\circ} \mathrm{C}$ (lit. (27) mp $\left.34^{\circ} \mathrm{C}\right) ;{ }^{1} \mathrm{H}$ NMR $\left(\mathrm{CDCl}_{3}\right) \& 8.28\left(\mathrm{~d}, J=1.8,1 \mathrm{H}, \mathrm{H}_{4}\right)$, $7.91\left(\mathrm{~d}, J=8.4,1 \mathrm{H}, \mathrm{H}_{6}\right), 7.85\left(\mathrm{dd}, J_{1}=1.8, J_{2}=8.4,1 \mathrm{H}\right.$, $\mathrm{H}_{5}$ ).

\section{3,4-Dibromo- $N, N$-diphenylbenzenesulfonamide (9)}

To a solution of 3,4-dibromobenzenesulfonyl chloride (2) $(1.0 \mathrm{~g}, 3.2 \mathrm{mmol})$ in dry $\mathrm{CHCl}_{3}(10 \mathrm{~mL})$ were added diphenylamine (3) $(0.7 \mathrm{~g}, 3.0 \mathrm{mmol})$ and pyridine $(0.5 \mathrm{~mL})$. The solution was refluxed for $14 \mathrm{~h}$, and cooled at room temperature, washed with aqueous $\mathrm{HCl}(5 \%, 10 \mathrm{~mL})$ and water until neutral. After drying over $\mathrm{MgSO}_{4}$ and evaporating the solvent, the crude product was recrystallized from ethanol to give colourless crystalline needles $(0.9$ g. $65 \%), \mathrm{mp}$ $160-161^{\circ} \mathrm{C}$; IR $\left(\mathrm{KBr} \mathrm{cm}^{-1}\right): 1957,1886,1589,1449,1446$, 1351; 'H NMR $\left(\mathrm{CDCl}_{3}\right) \delta .7 .93(\mathrm{~d}, J=2.0,1 \mathrm{H}), 7.74(\mathrm{~d}, J=$ $8.0,1 \mathrm{H}), 7.45\left(\mathrm{dd}, J_{1}=8.0, J_{2}=2.0,1 \mathrm{H}\right), 7.31(\mathrm{~m}, 10 \mathrm{H})$; MS ( $m / z$, relative intensity): $469\left(\mathrm{M}^{+}, 15\right), 467\left(\mathrm{M}^{+}, 30\right), 465$ $\left(\mathrm{M}^{+}, 14\right), 167$ (100). Anal. calcd. for $\mathrm{C}_{18} \mathrm{H}_{13} \mathrm{NBr}_{2} \mathrm{NO}_{2} \mathrm{~S}$ : C 46.25, H 2.78, N 3.00; found: C 46.41, H 2.66, N 2.96 .

\section{1-(3,4-Dibromophenylsulfonyl)pyrrole) (10)}

To freshly distilled pyrrole (4) $(220 \mathrm{mg}, 30 \mathrm{mmol})$ in dry THF $(5 \mathrm{~mL})$, cooled to $0^{\circ} \mathrm{C}$, was added $\mathrm{NaH}(300 \mathrm{mg}, 60 \%$ 
Scheme 2.<smiles>[R20]S(=O)(=O)c1ccc(Br)c(Br)c1</smiles>

$9 \mathrm{R}=\mathrm{Ph}$<smiles>[R]C1=CCC=C1</smiles><smiles>[Y16]=[R]=CC=C1C=CC=C1</smiles>

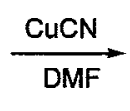<smiles>[R20]S(=O)(=O)c1ccc(C#N)c(C#N)c1</smiles>

$16 \mathrm{R}=\mathrm{Ph}$<smiles>[R2]C1=CCC=C1</smiles><smiles>[R18]=c1ccc2c(c1)C=CC=2</smiles>

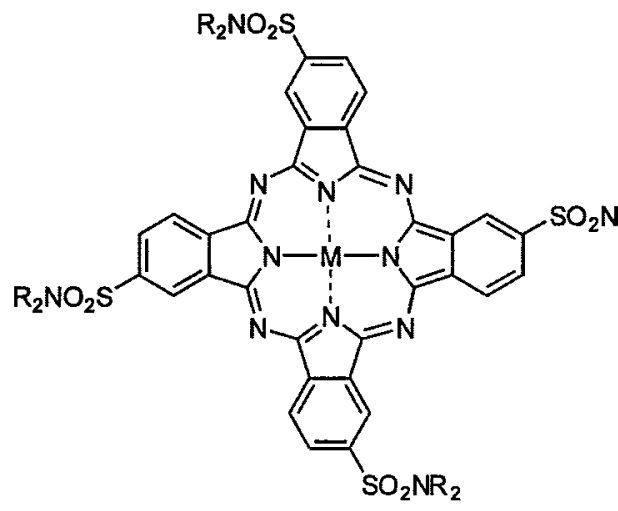

$19 \mathrm{R}=\mathrm{Ph}$

$M=2 \mathrm{H}$

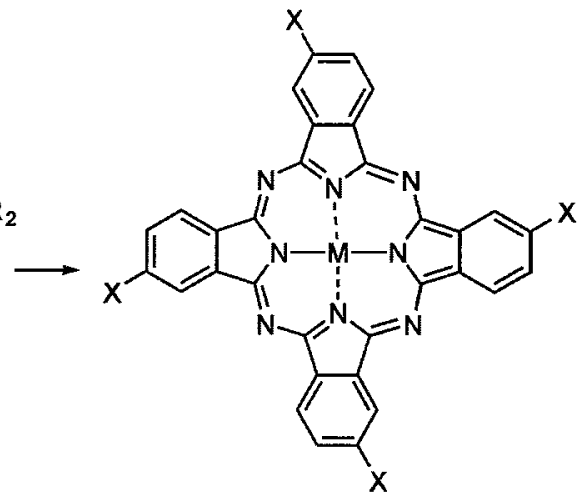<smiles>[R20]C1=CC=CC1</smiles><smiles>[R2][R]1ccc2cc[cH-]c2c1</smiles>

$M=2 H$<smiles>[R2][X]1=CCCC=C1</smiles>

$M=Z n$

$23 R$<smiles></smiles>

$M=Z n$

oil dispersion). 3,4-Dibromobenzenesulfonyl chloride (2) $(1.0 \mathrm{~g}, 3.2 \mathrm{mmol})$ was added over $30 \mathrm{~min}$. under Ar. After stirring at room temperature for $6 \mathrm{~h}$, the brown reaction mixture was carefully poured into a saturated $\mathrm{NH}_{4} \mathrm{Cl}$ solution $(30 \mathrm{~mL})$ placed in an ice bath. Ether $(30 \mathrm{~mL})$ was added and the aqueous layer was washed with ether $(20 \mathrm{~mL})$. The combined organic layer was washed with saturated $\mathrm{NH}_{4} \mathrm{Cl}$ until neutral. After drying over $\mathrm{MgSO}_{4}$ and evaporating the solvent, the crude product was recrystallized from ethanol to give colourless crystalline needles (545 $\mathrm{mg}, 50 \%$ ), $\mathrm{mp}$ $141-142^{\circ} \mathrm{C}$; IR $\left(\mathrm{KBr} \mathrm{cm}^{-1}\right): 3075(\mathrm{~s}, \mathrm{ArH}), 1375\left(\mathrm{~s}, \mathrm{SO}_{2} \mathrm{~N}\right)$, $1186\left(\mathrm{~s}, \mathrm{SO}_{2} \mathrm{~N}\right) ;{ }^{1} \mathrm{H} \mathrm{NMR}\left(\mathrm{CDCl}_{3}\right) \& 8.10(\mathrm{~d}, J=2.0,1 \mathrm{H})$, $7.77(\mathrm{~d}, J=8.4,1 \mathrm{H}), 7.63\left(\mathrm{dd}, J_{1}=8.4, J_{2}=2.0,1 \mathrm{H}\right), 7.16$ $(\mathrm{m}, 2 \mathrm{H}), 6.36(\mathrm{~m}, 2 \mathrm{H})$; MS $(\mathrm{m} / \mathrm{z}$, relative intensity): 367 $\left(\mathrm{M}^{+}, 50\right), 365\left(\mathrm{M}^{+}, 95\right), 363\left(\mathrm{M}^{+}, 50\right), 299(90), 235$ (100). Anal. calcd. for $\mathrm{C}_{10} \mathrm{H}_{7} \mathrm{NBr}_{2}$ : C 32.88, $\mathrm{H} \mathrm{1.92,} \mathrm{N} \mathrm{3.84;} \mathrm{found:}$ C $33.72, \mathrm{H} 1.87, \mathrm{~N} 3.91$.

1-(3,4-Dibromophenylsulphonyl)-2-ethylpyrrole (11)

The crude product, obtained as described for 10 but using 2-ethylpyrrole (5), recrystallized from ethanol to give in $3 \%$ yield fine colourless crystals $78-79^{\circ} \mathrm{C}$; IR $\left(\mathrm{KBr} \mathrm{cm}^{-1}\right): 3074$ $(\mathrm{s}, \mathrm{ArH}), 1372\left(\mathrm{~s}, \mathrm{SO}_{2} \mathrm{~N}\right), 1180\left(\mathrm{~s}, \mathrm{SO}_{2} \mathrm{~N}\right) ;{ }^{1} \mathrm{H} \mathrm{NMR}\left(\mathrm{CDCl}_{3}\right)$ $\delta: 8.14(\mathrm{~d}, J=1.6,1 \mathrm{H}), 8.03(\mathrm{~d}, J=7.8,1 \mathrm{H}), 7.73\left(\mathrm{dd}, J_{1}=\right.$ $\left.1.6, J_{2}=7.8,1 \mathrm{H}\right), 7.41(\mathrm{~d}, 1.7,1 \mathrm{H}), 6.31(\mathrm{t}, 1 \mathrm{H})(6.12 \mathrm{bs}$, $1 \mathrm{H}) ; 2.71(\mathrm{q}, J=7.3,2 \mathrm{H}), 1.24(\mathrm{t}, J=7.3,3 \mathrm{H}) ; \operatorname{MS}(\mathrm{m} / \mathrm{z}$, 
Scheme 3.<smiles>N#Cc1ccc(S(=O)(=O)n2cccc2)cc1C#N</smiles>

17

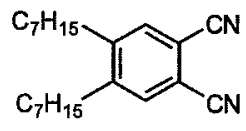

25

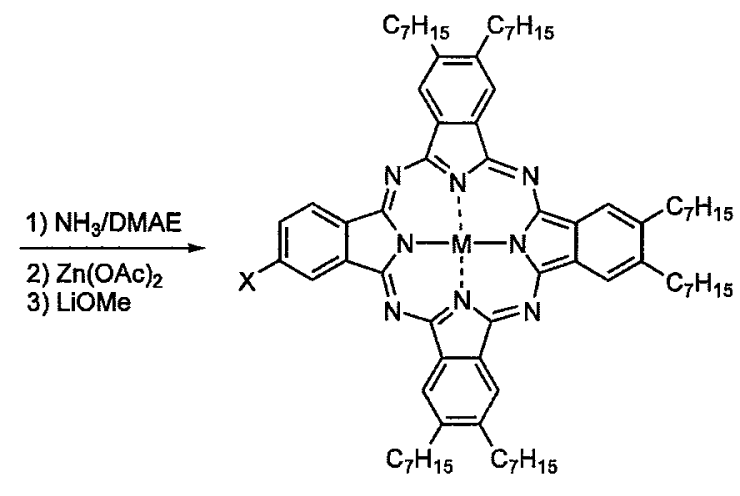

$26 \mathrm{M}=2 \mathrm{H}$<smiles>[Y]=[V]</smiles>

$27 \mathrm{M}=\mathrm{Zn}$<smiles>[Y]#[W]</smiles>

$28 \mathrm{M}=\mathrm{Zn}$

$\mathrm{X}=\mathrm{SO}_{3} \mathrm{Li}$

relative intensity): $393\left(\mathrm{M}^{+}, 75\right), 379(70), 315(67), 299$ (70), 80 (100). Anal. calcd. for $\mathrm{C}_{12} \mathrm{H}_{11} \mathrm{Br}_{2} \mathrm{NO}_{2} \mathrm{~S}: \mathrm{C} 36.64, \mathrm{H}$ 2.80, N 3.56; found: C 37.27, H 2.74, N 3.52.

\section{1-(3,4-Dibromophenylsulphonyl)-3,5-dimethylpyrazole} (12)

The crude product, obtained as described for $\mathbf{1 0}$ but using 3,5-dimethylpyrazole (6), was recrystallized from ethanol twice to give $0.98 \mathrm{~g}$ of 12 in $84 \%$ yield, $\mathrm{mp} 145-147^{\circ} \mathrm{C}$; IR $\left(\mathrm{KBr} \mathrm{cm}^{-1}\right): 3082(\mathrm{~s}, \mathrm{ArH}), 1374\left(\mathrm{~s}, \mathrm{SO}_{2} \mathrm{~N}\right), 1188\left(\mathrm{~s}, \mathrm{SO}_{2} \mathrm{~N}\right)$; ${ }^{1} \mathrm{H}$ NMR $\left(\mathrm{CDCl}_{3}\right) \& 8.19(\mathrm{~d}, J=1.5,1 \mathrm{H}), 7.75(\mathrm{~m}, 2 \mathrm{H})$, $5.94\left(\mathrm{~s},{ }^{1} \mathrm{H}\right), 2.51(\mathrm{~s}, 3 \mathrm{H}), 2.22(\mathrm{~s}, 31 \mathrm{H})$; MS $(\mathrm{m} / \mathrm{z}$, relative intensity): $394\left(\mathrm{M}^{+}, 10\right), 328(100), 299$ (30), 250 (40), 236 (50). Anal. calcd. for $\mathrm{C}_{11} \mathrm{H}_{10} \mathrm{Br}_{2} \mathrm{~N}_{2} \mathrm{O}_{2} \mathrm{~S}$ : C 33.50, H 2.54, N 7.11; found: C 33.72, H 2.30, N 7.14.

\section{1-(3,4-Dibromophenylsulfonyl)imidazole (13)}

To a solution of $(3)(1.0 \mathrm{~g}, 3.2 \mathrm{mmol})$ in a mixture of chloroform $(5 \mathrm{~mL})$ and methanol $(1 \mathrm{~mL})$ was added imidazole (7) in chloroform $(2 \mathrm{~mL})$ and $\mathrm{Na}_{2} \mathrm{CO}_{3}(1.0 \mathrm{~g})$. The mixture was stirred at room temperature for $22 \mathrm{~h}$, filtered, and the filtrate was washed with $\mathrm{HCl}(10 \%, 20 \mathrm{~mL})$ and water until neutral. After drying over $\mathrm{MgSO}_{4}$ and evaporating the solvent, the crude product was chromatographed with $40 \%$ ethyl acetate - hexane to give pure 13 as fine, colourless crystals (320 mg, $34 \%)$, mp $119-120^{\circ} \mathrm{C}$; IR $\left(\mathrm{KBr} \mathrm{cm}^{-1}\right)$ : $1938(\mathrm{~m}), 1768(\mathrm{~m}), 1564(\mathrm{~s}), 1464(\mathrm{~s}), 1381\left(\mathrm{~s}, \mathrm{SO}_{2} \mathrm{~N}\right) ;{ }^{1} \mathrm{H}$ NMR $\left(\mathrm{CDCl}_{3}\right) \& 8.19(\mathrm{~d}, J=2.0,1 \mathrm{H}), 8.04(\mathrm{~s}, 1 \mathrm{H}), 7.85(\mathrm{~d}$,

$J=8.0,1 \mathrm{H}), 7.73\left(\mathrm{dd}, J_{1}=8.0, J_{2}=2.0,1 \mathrm{H}\right), 7.32$ (brs, $1 \mathrm{H}) ; 7.16$ (brs, $1 \mathrm{H})$; MS $\left(\mathrm{m} / \mathrm{z}\right.$, relative intensity): $366\left(\mathrm{M}^{+}\right.$, 95) 299 (100), 235 (100). Anal. calcd. for $\mathrm{C}_{9} \mathrm{H}_{6} \mathrm{Br}_{2} \mathrm{~N}_{2} \mathrm{O}_{2} \mathrm{~S}: \mathrm{C}$ 29.51, H 1.64, N 7.65; found: C 29.61, H 1.46, N 7.61.

\section{1-(3,4-Dibromophenylsulfonyl)indole (14)}

Ground $\mathrm{NaOH}(2.0 \mathrm{~g})$ was mixed with tetrabutylammonium hydrogen sulfate $(140 \mathrm{mg})$ in $\mathrm{CH}_{2} \mathrm{Cl}_{2}(30 \mathrm{~mL})$. The reaction flask was cooled by a salt-ice bath to $0-4^{\circ} \mathrm{C}$ after which indole (8) (1.85 g, $15.8 \mathrm{mmol})$ was added. Sulfonyl chloride (7) $(5.0 \mathrm{~g}, 15.0 \mathrm{mmol})$ was added over $30 \mathrm{~min}$. and stirred for an additional $30 \mathrm{~min}$. in the ice bath. The solution was stirred at room temperature for $20 \mathrm{~h}$, washed with $\mathrm{HCl}$ $(10 \%, 20 \mathrm{~mL})$ and with water until neutral. After drying over $\mathrm{MgSO}_{4}$ and evaporating the solvent, the crude product was chromographed with $20 \%$ ethyl acetate - hexane to give 14 as a fine, colourless crystals $(3.1 \mathrm{~g}, 50 \% \mathrm{mp}) 125-126^{\circ} \mathrm{C}$; IR $\left(\mathrm{KBr} \mathrm{cm}{ }^{-1}\right): 3140,3081(\mathrm{~s}, \mathrm{ArH}), 1376\left(\mathrm{~s}, \mathrm{SO}_{2} \mathrm{~N}\right) ;{ }^{1} \mathrm{H}$ NMR $\left(\mathrm{CDCl}_{3}\right) \& 8.13(\mathrm{~d}, J=2.0,1 \mathrm{H}), 7.99(\mathrm{~d}, J=8.0,1 \mathrm{H})$, $7.66(\mathrm{~m}, 2 \mathrm{H}), 7.56(\mathrm{~m}, 2 \mathrm{H}), 7.37(\mathrm{~m}, 1 \mathrm{H}) ; 7.29(\mathrm{~m}, 1 \mathrm{H})$, $6.74(\mathrm{~d}, J=4.0,1 \mathrm{H})$; MS $\left(m / z\right.$, relative intensity): $417\left(\mathrm{M}^{+}\right.$, 45), $415\left(\mathrm{M}^{+}, 88\right) 413\left(\mathrm{M}^{+}, 43\right) 116$ (100). Anal. calcd. for $\mathrm{C}_{14} \mathrm{H}_{9} \mathrm{Br}_{2} \mathrm{NO}_{2} \mathrm{~S}$ : C 40.68, H 2.18, N 3.39; found: $\mathrm{C} 40.79, \mathrm{H}$ $1.95, \mathrm{~N} 3.37$.

\section{3,4-Dibromobenzenesulfonic acid (15)}

Compound 13 (50 mg, $0.14 \mathrm{mmol}$ ) was dissolved in methanol $(5 \mathrm{~mL})$ and $\mathrm{NaOMe}(40 \mathrm{mg}, 0.74 \mathrm{mmol})$ was added. 
The reaction solution was stirred at room temperature for $20 \mathrm{~h}$. The solvent was evaporated. The residue was dissolved in water $(50 \mathrm{~mL})$ and washed with ether $(10 \mathrm{~mL})$ followed by neutralization with aqueous $\mathrm{HCl}(36 \%)$. The solvent was evaporated, the residue recrystallized from water, and the product was further purified by ion-exchange resin (Dowex $50 \times 8-400)$. Recrystallization from EtOH (80\%) afforded pale, yellow needles of $15(26 \mathrm{mg}, 60 \%), \mathrm{mp} 64-66^{\circ} \mathrm{C}$ (lit. (27) $\left.\mathrm{mp} 66.5-67.5^{\circ} \mathrm{C}\right){ }^{1}{ }^{1} \mathrm{H}$ NMR $\left(\mathrm{D}_{2} \mathrm{O}\right) \delta: 7.99(\mathrm{~s}, 1 \mathrm{H}), 7.74$ $(\mathrm{d}, 1 \mathrm{H}), 7.52(\mathrm{dd}, 1 \mathrm{H})$. In a similar manner 12 gave 15 in $69 \%$ yield, while 10 and 14 were cleaved in methanol at reflux to give 15 in 72 and $57 \%$ yield, respectively.

\section{3,4-Dicyano- $N, N$-diphenylbenzenesulfonamide (16)}

To a solution of 9 (i.80 g, $3.87 \mathrm{mmol}$ ) in dry DMF $(20 \mathrm{~mL})$ was added $\mathrm{CuCN}(1.80 \mathrm{~g}, 20 \mathrm{mmol})$. The reaction solution was gently refluxed for $6 \mathrm{~h}$, cooled to room temperature, and $\mathrm{CH}_{2} \mathrm{Cl}_{2}(200 \mathrm{~mL})$ was added. A fine insoluble powder precipitated overnight. The crude product was purified by chromatography with $30 \%$ ethyl acetate - hexane as eluent to give colourless, scaly crystals ( $395 \mathrm{mg}, 29 \%), \mathrm{mp}$ $185-187^{\circ} \mathrm{C}$; IR $\left(\mathrm{KBr} \mathrm{cm}^{-1}\right): 3091$ (s, ArH), 2235, (s, CN), $1360\left(\mathrm{~s}, \mathrm{SO}_{2} \mathrm{~N}\right), 1186\left(\mathrm{~s}, \mathrm{SO}_{2} \mathrm{~N}\right) ;{ }^{1} \mathrm{H}$ NMR $\left(\mathrm{CDCl}_{3}\right) \& 7.90$ (s, $1 \mathrm{H}), 7.82(\mathrm{~d}, J=8.0,1 \mathrm{H}), 7.76(\mathrm{~d}, J=8.0), 7.19(\mathrm{~m}$, $5 \mathrm{H}), 7.07(\mathrm{~m}, 5 \mathrm{H})$; MS $\left(\mathrm{m} / \mathrm{z}\right.$, relative intensity): $359\left(\mathrm{M}^{+}\right.$, 60 ), 168 (100). Anal. calcd. for $\mathrm{C}_{20} \mathrm{H}_{13} \mathrm{~N}_{3} \mathrm{O}_{2} \mathrm{~S}$ : C 66.85, H 3.62, N 11.70; found: C $66.36 \mathrm{H} \mathrm{3.33,N} 11.56$.

\section{1-(3,4-Dicyanophenylsulfonyl)pyrrole (17)}

In a manner identical to that of 16,10 gave a crude product 17, which on flash chromatography with $30 \%$ ethyl acetate - hexanes as eluents yielded fine, colourless crystals (43\%), mp 155-157 ${ }^{\circ}$; IR $\left(\mathrm{KBr} \mathrm{cm}{ }^{-1}\right): 2235$ (s, CN), 1388, $1287\left(\mathrm{~s}, \mathrm{SO}_{2} \mathrm{~N}\right) ;{ }^{1} \mathrm{H}$ NMR $\left(\mathrm{CDCl}_{3}\right) \& 8.22(\mathrm{~d}, J=1.2,1 \mathrm{H})$, $8.17\left(\mathrm{dd}, J_{1}=1.2, J_{2}=8.2,1 \mathrm{H}\right), 7.97(\mathrm{~d}, J=8.2,1 \mathrm{H}), 7.16$ $(\mathrm{d}, J=1.8,2 \mathrm{H}), 6.41(\mathrm{~d}, J=1.8,2 \mathrm{H})$; MS $(m / z$, relative intensity): $257\left(\mathrm{M}^{+}, 100\right), 193$ (70). Anal. calcd. for $\mathrm{C}_{12} \mathrm{H}_{7} \mathrm{~N}_{3} \mathrm{O}_{2} \mathrm{~S}$ : C 56.03, H 2.72, N 16.34; found: $\mathrm{C} 56.17 \mathrm{H}$ 2.62, N 16.34.

\section{1-(3,4-Dicyanophenylsulfonyl)indole (18)}

As for 16 above, 14 gave a crude product, which on purification by flash chromatography with $30 \%$ ethyl acetate hexane as eluents, yielded pale yellow, fine crystals (45\%), mp 171-173 ${ }^{\circ} \mathrm{C}$; IR $\left(\mathrm{KBr} \mathrm{cm}{ }^{-1}\right): 3110$ (s, ArH), 2242 (s, $\mathrm{CN}), 1391\left(\mathrm{~s}, \mathrm{SO}_{2} \mathrm{~N}\right)$; 'H NMR $\left(\mathrm{CDCl}_{3}\right) \delta: 8.25\left(\mathrm{~s}, 1 \mathrm{H}, \mathrm{H}_{1}\right)$, $8.17\left(\mathrm{dd}, J_{1}=1.6, J_{2}=8.5,1 \mathrm{H}, \mathrm{H}_{3}\right), 7.96(\mathrm{~d}, J=8.5,1 \mathrm{H}$, $\left.\mathrm{H}_{2}\right), 7.88\left(\mathrm{~d}, J=8.0,1 \mathrm{H}, \mathrm{H}_{4}\right), 7.58\left(\mathrm{~d}, J=7.7,1 \mathrm{H}, \mathrm{H}_{7}\right), 7.51$ $\left(\mathrm{d}, J=3.5,1 \mathrm{H}, \mathrm{H}_{9}\right), 7.40\left(\mathrm{t}, J_{1}=8.0, J_{2}=7.3,1 \mathrm{H}, \mathrm{H}_{5}\right), 7.32$ $\left(\mathrm{t}, J_{1}=7.3, J_{2}=8.0,1 \mathrm{H}, \mathrm{H}_{6}\right), 6.79\left(\mathrm{~d}, J=3.5,1 \mathrm{H}, \mathrm{H}_{8}\right)$; MS ( $m / z$, relative intensity): $3.07\left(\mathrm{M}^{+}, 53\right), 116(52), 105(100)$. Anal. calcd. for $\mathrm{C}_{16} \mathrm{H}_{9} \mathrm{~N}_{3} \mathrm{O}_{2} \mathrm{~S}$ : $\mathrm{C} 62.54, \mathrm{H} \mathrm{2.93,} \mathrm{N} 1368$; found: C $62.95, \mathrm{H} 2.81, \mathrm{~N} 13.62$.

\section{$N, N, N, N^{\prime}, N^{\prime \prime}, N^{\prime \prime}, N^{\prime \prime \prime}, N^{\prime \prime \prime}$-Octaphenylphthalocyanine- \\ 2,9,16,23-tetrasulfonamide (19)}

Small pieces of metal lithium ( $30 \mathrm{mg}, 4.3 \mathrm{mmol}$ ) were dissolved in DMAE $(2 \mathrm{~mL})$ to which $16(100 \mathrm{mg}$, $0.28 \mathrm{mmol}$ ) was added. The reaction solution was stirred at $60-70^{\circ} \mathrm{C}$ (oil bath) for $20 \mathrm{~h}$, cooled to room temperature, and the sticky blue solution was poured into a mixture of ice
$(5 \mathrm{~g})$ and aqueous $\mathrm{HCl}(10 \mathrm{~mL}, 4 \mathrm{M})$. The precipitate was collected by filtration, and the crude product was purified using flash chromatography with $5 \%$ THF- $-\mathrm{CH}_{2} \mathrm{Cl}_{2}$ as eluent to give a dark blue, fine powder of $19(11 \mathrm{mg}, 11 \%), \mathrm{mp}$ $>310^{\circ} \mathrm{C}$. IR $\left.(\mathrm{KBr} \mathrm{cm})^{-1}\right): 1394,1164\left(\mathrm{~s}, \mathrm{SO}_{2} \mathrm{~N}\right)$; UV-vis (THF) $\left(\lambda_{\max }\right): 698,664,636,604,344,238 ;{ }^{1} \mathrm{H}$ NMR (pyridine- $d_{5}$ ): $8.91(\mathrm{~s}) 8.46(\mathrm{~s}), 7.85(\mathrm{~m}), 7.37(\mathrm{~m}), 6.96(\mathrm{~s})$; MS $\left(\mathrm{FAB}^{+}, m / z\right.$ relative intensity): $1438\left(\mathrm{M}^{+}\right)$. Anal. calcd. for $\mathrm{C}_{80} \mathrm{H}_{54} \mathrm{~N}_{12} \mathrm{O}_{8} \mathrm{~S}_{4}$ : C 66.76, H 3.75, N 11.68; found: $\mathrm{C}$ $66.95, \mathrm{H} 3.66, \mathrm{~N} 10.82$.

\section{2,9,16,23-Tetrakis(1-pyrrolysulfonyl)phthalocyanine (20)}

Compound 17 (200 mg, $0.78 \mathrm{mmol})$ was dissolved in $N, N$-dimethylaminoethanol (DMAE) $(2 \mathrm{~mL})$. As ammonia gas was bubbled through, the reaction mixture was heated to $100-110^{\circ} \mathrm{C}$ (sand bath) for $1 \mathrm{~h}$ and then refluxed for $4 \mathrm{~h}$. The reaction mixture was cooled to room temperature, the solvent evaporated, and water $(10 \mathrm{~mL})$ was added to the resulting sticky solution. The blue precipitate was collected by filtration or centrifugation and purified by flash chromatography using $5 \%$ THF $-\mathrm{CH}_{2} \mathrm{Cl}_{2}$ as eluent to give in the first fraction phthalocyanine 20 as dark blue crystals $(66 \mathrm{mg}$, $33 \%), \mathrm{mp}>310^{\circ} \mathrm{C}$; IR $\left(\mathrm{KBr} \mathrm{cm}^{-1}\right): 3141(\mathrm{~m}, \mathrm{ArH}), 1374$ (s, $\left.\mathrm{SO}_{2} \mathrm{~N}\right), 1191\left(\mathrm{~s}, \mathrm{SO}_{2} \mathrm{~N}\right.$ ); UV-vis (THF), $\left(\lambda_{\max }\right): 696,662$, $634,608,394,348$; ${ }^{1} \mathrm{H}$ NMR (THF- $\left.d_{8}\right) \delta: 9.40,9.38,8.45$ $\left(\mathrm{m}, 4 \mathrm{H}, \mathrm{H}_{3}\right), 7.79\left(\mathrm{~m}, 4 \mathrm{H}, \mathrm{H}_{4}\right), 6.95,5.87\left(\mathrm{~m}, 8 \mathrm{H}, \mathrm{H}_{1}\right), 6.93$ $\left(\mathrm{m}, 8 \mathrm{H}, \mathrm{H}_{2}\right), 664\left(\mathrm{~m}, 4 \mathrm{H}, \mathrm{H}_{5}\right)$; MS $\left(\mathrm{FAB}^{+}, \mathrm{m} / \mathrm{z}\right.$, relative intensity): $1030\left(\mathrm{M}^{+}\right)$. Anal. calcd. for $\mathrm{C}_{48} \mathrm{H}_{130} \mathrm{~N}_{12} \mathrm{O}_{8} \mathrm{~S}_{4}: \mathrm{C}$ 55.92, H 2.91, N 16.31; found: C 55.80, H 2.90, N 15.78 .

2,9,16,23-Tetrakis(1-indolylsulfonyl)phthalocyanine (21)

As for 20, but from 18, phthalocyanine 21 was obtained as dark, blue crystals in $42 \%$ yield, $\mathrm{mp}>310^{\circ} \mathrm{C}$; IR $\left(\mathrm{KBr} \mathrm{cm}^{-1}\right): 1387\left(\mathrm{~s}, \mathrm{SO}_{2} \mathrm{~N}\right) 1173\left(\mathrm{~s}, \mathrm{SO}_{2} \mathrm{~N}\right)$; UV-vis (THF) $\left(\lambda_{\max }\right): 698,666,634,612,346,248 ;{ }^{1} \mathrm{H}$ NMR $\left(\mathrm{CDCl}_{3}\right) \delta$ : $9.56(\mathrm{~m}, \mathrm{br}), 8.88(\mathrm{~m}, \mathrm{br}), 8.70(\mathrm{~m}, \mathrm{br}), 8.54(\mathrm{~m}), 8.14(\mathrm{~m})$, $8.03(\mathrm{~m}), 7.67(\mathrm{~m}), 7.55(\mathrm{~m}), 7.01(\mathrm{~m}), 6.92(\mathrm{~m})$; MS (FAB $m / s$, relative intensity): $1230\left(\mathrm{M}^{+}, 40\right), 671(70), 539(100)$. Anal. calcd. for $\mathrm{C}_{64} \mathrm{H}_{38} \mathrm{~N}_{12} \mathrm{O}_{8} \mathrm{~S}_{4}: \mathrm{C} 62.44, \mathrm{H}, 3.09, \mathrm{~N} 13.66$; found: C 62.85, H 3.32, N 13.27.

\section{2,9,16,23-Tetrakis(1-pyrrolylsulfonyl)phthalocyanine zinc(II) (22)}

Compound 17 (200 $\mathrm{mg}, 0.78 \mathrm{mmol})$ was dissolved in $2-N, N$-dimethylaminoethanol (DMAE) $(2 \mathrm{~mL})$ containing anhydrous $\mathrm{Zn}(\mathrm{OAc})_{2}(200 \mathrm{mg})$. The reaction mixture was heated to $100-110^{\circ} \mathrm{C}$ (sand bath) and kept at that temperature for $1 \mathrm{~h}$ while ammonia gas was bubbled through it. The solution was refluxed for $4 \mathrm{~h}$ and cooled to room temperature. The solvent was evaporated and water $(10 \mathrm{~mL})$ was added to the resulting sticky solution. The blue precipitate was collected by filtration or centrifugation and purified by flash column chromatography using $50 \% \mathrm{THF}-\mathrm{CH}_{2} \mathrm{Cl}_{2}$ as eluent to give in the first fraction 22 as dark crystals $(68 \mathrm{mg}$, $32 \%), \mathrm{mp}>310^{\circ} \mathrm{C}$; IR $\left(\mathrm{KBr} \mathrm{cm}^{-1}\right): 1371\left(\mathrm{~m}, \mathrm{SO}_{2} \mathrm{~N}\right) 1167(\mathrm{~s}$, $\left.\mathrm{SO}_{2} \mathrm{~N}\right)$; UV-vis (THF) $\left(\lambda_{\text {max }}\right): 672,610,350,242 ;{ }^{\prime} \mathrm{H}$ NMR $\left(\mathrm{THF}-d_{8}\right) \& 9.73(\mathrm{br}), 7.36-8.66(\mathrm{~m}, \mathrm{br}), 6.59-6.90(\mathrm{~m}, \mathrm{br})$. MS $\left(\mathrm{FAB}^{\dagger}, m / z\right.$, relative intensity): $1093\left(\mathrm{M}^{+}+1250\right), 964$ (20), $625 \quad$ (52) 558 (100). Anal. calcd. for $\mathrm{C}_{48} \mathrm{H}_{128} \mathrm{~N}_{12} \mathrm{O}_{8} \mathrm{~S}_{4} \mathrm{Zn}: \mathrm{C} 52.70, \mathrm{H} 2.56, \mathrm{~N} 15.37$; found: $\mathrm{C}$ $52.29, \mathrm{H} 2.80$, N 14.46 . 


\section{2,9,16,23-Tetrakis(1-indolylsulfonyl)phthalocyanine zinc(II) (23)}

As for 22, 18 yielded a crude product which was purified by flash chromatography by using $40 \%$ THF-benzene as eluent to give 23 as dark blue crystals $(68 \%), \mathrm{mp}>310^{\circ} \mathrm{C}$; IR $\left(\mathrm{KBr} \mathrm{cm}{ }^{-1}\right): 1376\left(\mathrm{~m}, \mathrm{SO}_{2} \mathrm{~N}\right), 1173\left(\mathrm{~s}, \mathrm{SO}_{2} \mathrm{~N}\right)$; UV-vis (THF) $\left(\lambda_{\text {max }}\right): 676,610,360,244 ;{ }^{1} \mathrm{H} \mathrm{NMR}\left(\mathrm{CDCl}_{3}\right) \& 9.79$ $(\mathrm{m}), 9.12(\mathrm{~m}), 8.68(\mathrm{~m}), 8.31(\mathrm{~m}), 8.04(\mathrm{~m}), 7.60(\mathrm{~m}), 7.17$ $(\mathrm{m}), 6.86(\mathrm{~m}), 6.62(\mathrm{~m})$; MS (FAB,$+ \mathrm{m} / \mathrm{z}$, relative intensity): $1293\left(\mathrm{M}^{i}, 60\right), 1113(50), 919$ (100). Anal. calcd. for $\mathrm{C}_{64} \mathrm{H}_{36} \mathrm{~N}_{12} \mathrm{O}_{8} \mathrm{~S}_{4} \mathrm{Zn}$ : C 59.40, H 2.78, N 12.99; found: C 59.19; H 3.09, N 12.65 .

\section{Phthalocyanine-2,9,16,23-tetrasulfonic acid (24)}

\section{Method A}

Small pieces of metal lithium ( $400 \mathrm{mg}, 58 \mathrm{mmol}$ ) were dissolved in DMAE (20 mL) and 20 (100 mg, $0.097 \mathrm{mmol})$ was added. The solution was stirred at $60-70^{\circ} \mathrm{C}$ for $30 \mathrm{~h}$. An aliquot was taken and added to a mixture of water and $\mathrm{CH}_{2} \mathrm{Cl}_{2}$. The organic solution appeared colourless. The reaction mixture was cooled to room temperature, water was added, and the solvent was reduced to half volume under vacuum. After acetone $(15 \mathrm{~mL})$ was added, the precipitates were separated by centrifugation and washed with aqueous $\mathrm{HCl}(36 \%, 5 \times 3 \mathrm{~mL})$. Drying under high vacuum gave a dark blue powder of $24(25,31)(70 \mathrm{mg}, 86 \%), \mathrm{mp}>310^{\circ} \mathrm{C}$; IR $\left(\mathrm{KBr} \mathrm{cm}{ }^{-1}\right): 3400\left(\mathrm{~s}, \mathrm{SO}_{3} \mathrm{H}\right), 1472\left(\mathrm{~s}, \mathrm{SO}_{3} \mathrm{H}\right)$; UV-vis $(\mathrm{MeOH})\left(\lambda_{\max }\right): 692,656 ;{ }^{1} \mathrm{H}$ NMR (DMSO- $\left.d_{6}\right) \& 10.19(\mathrm{br}$, $4 \mathrm{H}), 9.71(\mathrm{br}, 4 \mathrm{H}), 9.53(\mathrm{~m}, 4 \mathrm{H}), 8.60(\mathrm{~m}, 4 \mathrm{H})$; MS (electrospray, $m / z): 834.13(\mathrm{M}+1-\mathrm{H})^{-}, 753.50(\mathrm{M}+1-$ $\left.\mathrm{H}-\mathrm{SO}_{3} \mathrm{H}\right)^{-}, 416.11(\mathrm{M}-2 \mathrm{H})^{2-}, 277.07(\mathrm{M}-3 \mathrm{H})^{3-}, 250.19$ $\left(\mathrm{M}+\mathrm{I}-\mathrm{SO}_{3} \mathrm{H}-3 \mathrm{H}\right)^{3-}, 207.49(\mathrm{M}-4 \mathrm{H})^{4-}$.

\section{Method B}

Small pieces of metal lithium ( $40 \mathrm{mg}, 5.8 \mathrm{mmol}$ ) were dissolved in a mixture of methanol $(2 \mathrm{~mL})$ and THF $(2 \mathrm{~mL})$ to which $21(20 \mathrm{mg})$ was added. The solution was refluxed for $20 \mathrm{~h}$. TLC with $5 \%$ THF-- $\mathrm{CH}_{2} \mathrm{Cl}_{2}$ as eluent showed that the starting material had disappeared. The solvent was evaporated and the residue was washed with aqueous $\mathrm{HCl}(36 \%$, $5 \times 3 \mathrm{~mL}$ ) to give a product identical with that from method A (23 mg, 84\%).

\section{9,10,16,17,23,24-Hexakis-(1-heptyl)-2-(1-}

pyrrolylsulfonyl)phthalocyanine (26)

Excess 4,5-diheptylphthalonitrile (25) (29), prepared by our previously described method (32), (200 $\mathrm{mg}, 0.69 \mathrm{mmol}$ ) and $17(20 \mathrm{mg}, 0.078 \mathrm{mmol})$ were dissolved in DMAE $(3 \mathrm{~mL})$. As ammonia gas was bubbled through, the reaction mixture was heated to $100-110^{\circ} \mathrm{C}$ (sand bath). After $1 \mathrm{~h}$, the solution was raised to reflux temperatures and refluxed for $20 \mathrm{~h}$. The reaction mixture was cooled to room temperature, ice $(5 \mathrm{~g})$ was added to the resulting sticky solution, and the precipitates were separated by centrifugation followed by sequential washing with water $(10 \times 2 \mathrm{~mL})$ and methanol $(10$ $\times 2 \mathrm{~mL}$ ). The blue precipitate was collected by filtration or centrifugation and purified by flash column chromatography using $\mathrm{CH}_{2} \mathrm{Cl}_{2}$ as eluent. After evaporation of the solvent, the residue was rechromatographed with $10 \%$ EtOAc-hexanes and then $5 \% \mathrm{THF}-\mathrm{CH}_{2} \mathrm{Cl}_{2}$ as eluents. The first fraction con- tained two spots, which was rechromatrographed later. The second fraction was a mixture of multisulfonate Pcs $(8.7 \mathrm{mg})$. The first fraction was separated by column chromatography using $50 \% \mathrm{CH}_{2} \mathrm{Cl}_{2}$-hexanes to give $26(4.5 \mathrm{mg}, 4.7 \%)$, mp $>310^{\circ} \mathrm{C}$; IR $\left(\mathrm{KBr} \mathrm{cm}^{-1}\right): 1337\left(\mathrm{~m}, \mathrm{SO}_{2} \mathrm{~N}\right), 1167\left(\mathrm{~s}, \mathrm{SO}_{2} \mathrm{~N}\right)$; UV-vis (THF) $\left(\lambda_{\max }\right): 714,692,686,656 ;{ }^{1} \mathrm{H}$ NMR $\left(\mathrm{CDCl}_{3}\right)$ $\delta: 9.54(\mathrm{~s}, 1 \mathrm{H}), 9.03(\mathrm{~s}, 1 \mathrm{H}), 8.71(\mathrm{~m} 3 \mathrm{H}), 8.50(\mathrm{~m}, 2 \mathrm{H}), 8.25$ $(\mathrm{d}, J=7.6,1 \mathrm{H}), 7.69(\mathrm{~s}, 1 \mathrm{H}), 7.57(\mathrm{~s}, \mathrm{Py}-\mathrm{H}, 2 \mathrm{H}), 6.42(\mathrm{~s}$, Py-H, 2H), $3.15(\mathrm{~m}, 12 \mathrm{H}), 1.49(\mathrm{~m}, 78 \mathrm{H})$; MS (FAB $+m / z)$ : $1232\left(\mathrm{M}^{+}+1\right)$. Anal. calcd. for $\mathrm{C}_{78} \mathrm{H}_{105} \mathrm{~N}_{9} \mathrm{O}_{2} \mathrm{~S}: \mathrm{C} 76.04, \mathrm{H}$ 8.53, N 10.24; found: C 76.62, H 8.85, N 9.52.

\section{9,10,16,17,23,24-Hexakis-(1-heptyl)-2-}

(1-pyrrolylsulfonyl)-phthalocyanine zinc(II) (27)

Metal-free Pc 26 (10 mg, $0.008 \mathrm{mmol})$ was dissolved in a mixture of toluene $(3 \mathrm{~mL})$ and DMF $(3 \mathrm{~mL})$ to which anhydrous $\mathrm{Zn}(\mathrm{OAc})_{2}$ was added. The reaction solution was refluxed for $24 \mathrm{~h}$, the solvent evaporated, and the residue washed with water $(10 \mathrm{~mL} \times 2)$ and methanol $(10 \mathrm{~mL} \times 2)$. After drying under vacuum, the crude product was purified by column chromatography using $5 \% \mathrm{THF}-\mathrm{CH}_{2} \mathrm{Cl}_{2}$ as eluent to give 27 as a dark blue powder $(9 \mathrm{mg}, 86 \%), \mathrm{mp}>310^{\circ} \mathrm{C}$; IR $\left(\mathrm{KBr} \mathrm{cm}{ }^{-1}\right)$ : $1339,1166\left(\mathrm{~s}, \mathrm{SO}_{2} \mathrm{~N}\right)$; UV-vis (THF) $\left(\lambda_{\max }\right)$ : $692,666,634,604,354 ;{ }^{1} \mathrm{H}$ NMR $\left(\mathrm{C}_{6} \mathrm{D}_{6}\right) \& 9.96(\mathrm{br}, 1 \mathrm{H})$, 9.10-8.12 (m, 8H), 7.45 (py-H, 2H), 6.13 (py-H, 2H), 3.15 $(\mathrm{m}, 12 \mathrm{H}), 1.70(\mathrm{~m}, 60 \mathrm{H}), 1.01(\mathrm{~m}, 18 \mathrm{H})$; MS (FABt, $\mathrm{m} / \mathrm{z}$, relative intensity): $1295\left(\mathrm{M}^{+}+1,100\right), 736(60)$. Anal. calcd. for $\mathrm{C}_{78} \mathrm{H}_{103} \mathrm{~N}_{9} \mathrm{O}_{2} \mathrm{SZn}$ : C 72.33, $\mathrm{H} 7.96, \mathrm{~N}$ 9.74; found: C $72.12, \mathrm{H} 8.21, \mathrm{~N} 9.14$.

\section{Lithium 9,10,16,17,23,24-hexakis-(1-}

heptyl)phthalocyanine-2-sulfonate zinc(II) (28)

To a solution of lithium $(40 \mathrm{mg})$ in $\mathrm{MeOH}(2 \mathrm{~mL})$ was added $27(17 \mathrm{mg})$. The solution was refluxed for $24 \mathrm{~h}$, the solvent evaporated, and the crude product purified by column chromatography (Si-gel, 70-230 mesh) using 40\% EtOAc-hexanes and methanol as eluents to give 28 as a dark blue fine powder $(14 \mathrm{mg}, 85 \%), \mathrm{mp}>310^{\circ} \mathrm{C}$; IR $\left(\mathrm{KBr} \mathrm{cm}^{-1}\right)$ : $1110\left(\mathrm{~s}, \mathrm{SO}_{3} \mathrm{Li}\right)$. UV-vis (THF) $\left(\lambda_{\max }\right): 676,616,346,240$ $(0.47)$; ' $\mathrm{l} H$ NMR (DMSO- $\left.d_{6}\right) \delta 9.60(\mathrm{br}, 1 \mathrm{H}), 9.31(\mathrm{~d}, J=$ $8.0,1 \mathrm{H}), 9.15(\mathrm{~m}, 6 \mathrm{H}), 8.42(\mathrm{~d}, J=8.0,1 \mathrm{H}), 1.94(\mathrm{~m}, 6 \mathrm{H})$, $1.38(\mathrm{~m}, 60 \mathrm{H}), 0.92(\mathrm{~m}, \mathrm{br}, 18 \mathrm{H})$; MS (FAB,$+ \mathrm{m} / \mathrm{z}$, relative intensity): $1251\left(\mathrm{M}^{+}, 100\right)$.

\section{Acknowledgement}

We are grateful to the Natural Sciences and Engineering Research Council of Canada for financial support of this research.

\section{References}

1. R.W. Boyle and D. Dolphin. Photochem. Photobiol. 64, 469 (1996); T.J. Dougherty. Photochem. Photobiol. 58, 895 (1993).

2. A.M. Fisher, A.L. Murphree, and C.J. Gamer. Lasers Surg. Med. 17, 2 (1995).

3. C.J. Byrne, L.V. Marshallsay, S.Y. Sek, and A.P. Ward. In Photodynamic therapy of neoplastic disease. Edited by D. Kessel. CRC press, Boca Raton. 1990. pp. 133-144.

4. (a) J.D. Spikes. Photochem Photobiol. 43, 691 (1986); (b) J.E. van Lier. In Photodynamic therapy of neoplastic disease. 
Edited by D. Kessel. CRC Press, Boca Raton. 1990. pp. 279-290.

5. W.S. Chan, J.F. Marshall, R. Svensen, J. Bedwell, and I.R. Hart. Cancer Res. 50, 4533 (1990).

6. N. Brasseur, H. Ali, R. Langlois, and J.E. van Lier. Photochem. Photobiol. 46, 739 (1987).

7. B. Paquette, H. Ali, R. Langlois, and J.E. van Lier. Photochem. Photobiol. 47, 215 (1988).

8. H. Ali, R. Langlois, J.R. Wagner, N. Brasseur, B. Paquette, and J.E. van Lier: Photochem. Photobiol. 47, 713 (1988).

9. P. Margaron, P. Madarnas, R. Ouellet, and J.E. van Lier. Anticancer Res. 16, 613 (1996).

10. W.-S. Chan, N. Brasseur, C. La Madeleine, and J.E. van Lier. Anticancer Res. 16, 1998 (1996).

11. S.V. Kudrevich, H. Ali, and J.E. van Lier. J. Chem. Soc. Perkin Trans. 1, 2767 (1994).

12. S.V. Kudrevich, S. Gilbert, and J.E. van Lier. J. Med. Chem. 40, 3897 (1997).

13. S.V. Kudrevich, N. Brasseur, C. La Madeleine, S. Gilbert, and J.E. van Lier. J. Med. Chen. 40, 3897 (1997).

14. N. Kobayashi, R. Kondo, S. Nakajima, and T. Osa. J. Am. Chem. Soc. 112, 9640 (1990).

15. A. Weitemeyer, H. Kliesch, and D. Wöhrle. J. Org. Chem. 60, 4900 (1995); J. Rauschnabel and M. Hanack. Tetrahedron Lett. 36, 1629 (1995).

16. S. Searles and S. Nukina. Chem. Rev, 59, 1077 (1959).

17. E. Vedejs and S. Lin. J. Org. Chem. 59, 1602 (1994); C. Goulaouic-Dubois, A. Guggisberg, and M. Hesse. J. Org. Chem. 60, 5969 (1995).

18. J.E. Richmond and T.J. Atkins. J. Am. Chem. Soc. 96, 2268 (1974); R.C. Roemmele and H. Rapoport. J. Org. Chem. 53, 2367 (1988).

19. S. Ji, L.B. Gorther, A. Waring, A. Battisti, S. Bank, W.D. Closson, and P. Wriede. J. Am. Chem. Soc. 89, 5311 (1967);
B. Nyasse, L. Grehn, and U. Ragnarsson. J. Chem. Soc. Chem. Commun. 1017 (1997).

20. J.C. Roberts, H. Gao, A. Gopalsamy, A. Kongsjahju, and R.J. Patch. Tetrahedron Lett. 38, 355 (1997).

21. J.J. Plattner, P.A. Marcotte, H.D. Kleinert, H.H. Stein, J. Greer, G. Bolis, A.K.L. Fung, B.A. Bopp, J.R. Luly, H.L. Sham. D.J. Kempf, S.H. Rosenberg, J.F. Dullaria, B. De, I. Merits, and T.J. Perun. J. Med. Chem. 31, 2277 (1988).

22. R.J. Sundberg. J. Heterocyclic Chem. 14, 517 (1977).

23. (a) E.P. Papadopoulos and N.F. Haidar. Tetrahedron Lett. 1721 (1968); (b) M. Kakushima, P. Hamel, R. Frenette, and J. Rokach. J. Org. Chem. 48, 3214 (1983); (c) H.J. Gogan, R. McDonald, and L.G. Edwards. Can. J. Chem. 63, 896 (1985).

24. (a) V.D. Illi. Synthesis, 136 (1979); (b) D.M. Ketcha and G.W. Gribble. J. Org. Chem. 50, 5451 (1985); J.-Y Mérour, B. Malapel, and E. Desarbe. Synth. Commun. 26, 3267 (1996).

25. L.I. Solov'eva, S.A. Mikhalenko, E.V. Chernykh, and E.A Luk'yanets. Zh. Obshch. Khim. 52, 90 (1982).

26. (a) Ellis and Romney-Alexander. Chem. Rev. 87, 779 (1987); (b) M. Hu, N. Brasseur, S.Z. Yildiz, J.E. van Lier, and C.C. Leznoff. J. Med. Chem. 41, 1789 (1998).

27. (a) C. Goslich. Ann. 186, 148 (1877); (b) L. Spiegelberg. Ann, 197, 257 (1879).

28. C.C. Leznoff, M. Hu, and K.J.M. Nolan. J. Chem. Soc. Chem. Commun. 1245 (1996).

29. H. Mishi, N. Azuma, and K. Kitahara. J. Heterocycl. Chem. 29, 475 (1992).

30. V.M. Negrimousky, V.M. Derkacheva, E.A. Luk'yanets, A. Weitemeyer, D. Wöhrle, and G. Schneider. Phosphorus Sulfur Silicon Relat. Elem. 104, 161 (1995).

31. R.P. Linstead and F.T. Weiss. J. Chem. Soc. 2975 (1950).

32. D.S. Terekhov, K.J.M. Nolan, C.R. McArthur, and C.C. Leznoff. J. Org. Chem. 61, 3034 (1996). 